\title{
A LOS DOS LADOS DEL ATLÁNTICO. RECONFIGURACIONES DE LOS PROYECTOS MIGRATORIOS Y LA VIDA FAMILIAR TRANSNACIONAL DE LA POBLACIÓN COLOMBIANA EN ESPAÑA
}

\section{Both sides of the Atlantic. Migratory projects reconfiguration and Colombian population in Spain transnational family life}

\author{
María Margarita Echeverri Buriticá* \\ * Profesora Asociada, Facultad de Psicología, Pontificia Universidad Javeriana, Bogotá - \\ Colombia. Integrante GIIM. \\ mariamargaritaecheverri@gmail.com
}

Palabras
clave
Migración
familiar
Enfoque
transnacional
Género
Colombia
España

Keywords

Family migration Transnational focus gender generational Colombia Spain

\begin{abstract}
Resumen
Desde un enfoque transnacional, de género y generacional este artículo se ropone reconstruir las transformaciones de los proyectos migratorios y de las formas de organización de la vida familiar de los migrantes colombianos asentados en territorio español, desde finales de los años noventa hasta el inicio de la segunda década del siglo XXI. Se analizará la incidencia de tres factores/momentos específicos: el incremento de la migración femenina desde Colombia hacia España a finales de la década de los años noventa, el visado impuesto a la migración colombiana en el año 2002 y el impacto de la actual crisis económica a partir del año 2007. El análisis se sustenta en un trabajo de campo longitudinal en lugares de origen y destino, desarrollado con las mismas familias durante más de diez años, prestando especial atención a los integrantes jóvenes.
\end{abstract}

\section{Abstract}

From a transnational, gender and generational focus, this article proposes rebuilding migratory projects transformation and the organizational family living ways of the Colombian migrants settled in Spain, from the late nineties to the second decade of the 21st century. The incidence of three specific factors/moments will be analyzed: the women migration from Colombia to Spain at the end of the nineties, the Visa requirements imposed to Colombian citizens in 2002 and the impact of the actual economic crisis. The analysis is based on longitudinal fieldwork in origin and destination places, developed with the same families during over ten years, paying special attention to the young population.

Echeverri Buriticá, M., 2014, "A los dos lados del Atlántico. Reconfiguraciones de los proyectos migratorios y la vida familiar transnacional de la población colombiana en España", en Papeles del CEIC, vol. 2014/2, no 109, CEIC (Centro de Estudios sobre la Identidad Colectiva), Universidad del País Vasco, http://dx.doi.org/10.1387/pceic.12988 


\section{INTRODUCCIÓN}

Hasta hace poco tiempo, en España los proyectos migratorios familiares habían recibido poca atención desde el campo de estudio de las migraciones, sobre todo en lo que respecta a las trayectorias de las generaciones jóvenes de las familias migrantes. Sin embargo, desde finales del siglo $X X$, la significativa presencia de las mujeres en las corrientes migratorias latinoamericanas hacia territorio español, especialmente de República Dominicana, Ecuador y Colombia, y, más recientemente, Bolivia, Brasil y Paraguay- ha sacado a la luz dinámicas familiares complejas que han propiciado los debates sobre el binomio migración y familia. La feminización de las migraciones latinoamericanas hacia España ha generado cambios estructurales y simbólicos en las relaciones de género y generacionales al interior de las familias, donde se han evidenciado nuevas negociaciones alrededor de las prácticas de la maternidad y la paternidad (Pedone, 2006; Medina Villegas, 2009), la reedición de los múltiples vínculos y los cambios en la organización del cuidado en un contexto transnacional (Pedone, 2010).

De esta manera, la introducción de los análisis sobre las redes migratorias desde la perspectiva transnacional y con enfoque de género y generacional en el campo de las migraciones, potenció la mirada hacía los procesos migratorios familiares y visibilizó la participación de los diferentes miembros de las familias como actores claves en el trazado de las trayectorias migratorias (Gregorio Gil, 1998; Pedone, 2006 y 2008; Escrivá y Ribas, 2004; Gregorio Gil y Ramírez, 2000; Suárez Navas, 2004). Emergieron así, los niños, niñas y jóvenes como actores fundamentales en el juego de las relaciones de poder al interior de los grupos familiares. Algunos estudios incorporaron la perspectiva transnacional para estudiar las trayectorias de inserción y estrategias de pertenencia de las generaciones jóvenes de las familias migrantes (García Borrego, 2008; Echeverri, 2005 y 2010; Pedreño y Castellanos, 2010).

Para el caso de Colombia, si bien los procesos de migración internacional se revelaron como proyectos familiares desde finales de la década de los años noventa, cuando comienza a intensificarse la corriente migratoria hacia España, el interés académico por la migración familiar es reciente. Entre los estudios pioneros sobre las dinámicas familiares y de género en el contexto de la migración destacan los trabajos sobre vínculos parentales (Micolta, 2007), la maternidad transnacional (Puyana, Motoa y Viviel, 2009; Rivas y Gonzálvez, 2009; 
Medina Villegas, 2009; Santos Pérez, 2011) y la organización transnacional del cuidado (Gonzálvez, 2010; Puyana, Micolta y Palacio, 2013). También existen una minoría de trabajos que han puesto el acento en los y las jóvenes como actores sociales fundamentales de las dinámicas familiares en un contexto migratorio (Carrasquilla y Echeverri, 2003; Echeverri, 2005 y 2010).

Desde inicios del siglo XXI la migración por motivos familiares es la principal vía de ingreso de la población colombiana a España, superando a las solicitudes de asilo (Sanabria, 2008), pero es significativa la falta de estudios sobre los proceso de reunificación familiar y más aún sobre la incidencia de las políticas y regulaciones migratorias en la vida familiar de la población migrante. Una excepción son las investigaciones realizadas por las integrantes del Grupo Interdisciplinario de Investigadoras Migrantes (GIIM), quienes han centrado la atención en las relaciones entre la política migratoria española y las formas de organización familiar de migrantes colombianos entre Colombia y España (Pedone, Echeverri y Gil Araujo, en prensa).

Estos antecedentes muestran que el trazado de los proyectos migratorios de los jóvenes colombianos y sus familias se han visto fuertemente influenciados por factores particulares de los contextos migratorios actuales, que en su interrelación y dinámica cambiante en el marco del orden internacional y la globalización, aparecen como determinantes en la definición de sus trayectorias: la feminización de las migraciones, las restricciones políticas y el cierre de fronteras, y la crisis financiera y económica actual. En esta línea, la aceleración, politización, diferenciación y feminización son los elementos más relevantes de los actuales movimientos migratorios (Castles y Miller, 2007).

En este marco, desde un enfoque transnacional, de género y generacional este artículo se propone reconstruir las transformaciones de los proyectos migratorios familiares y de las trayectorias de la vida familiar de los migrantes colombianos asentados en territorio español, desde finales de los años noventa hasta el inicio de la segunda década del siglo XXI, prestando especial atención a la incidencia de tres factores y momentos específicos que han marcado sus trayectorias: el incremento de la migración femenina desde Colombia hacia España a finales de las años noventa, el visado impuesto a la migración colombiana en el año 2002 y el impacto de la actual crisis económica a partir de 2007. 
El análisis se basa en trabajos de campo longitudinales realizados en varios proyectos de investigación llevados a cabo por la autora. En primer lugar, se desarrolló la tesis doctoral "Son diez horas de viaje y cinco años que te meten encima". Proyectos, identidades y vínculos transnacionales de los y las jóvenes colombianas en España, una investigación cualitativa, etnográfica y longitudinal, desarrollada entre 2001 y 2007 con 65 jóvenes colombianos, hombres y mujeres, que migraron por diferentes motivos (reagrupación familiar, autónomamente o por exilio) y sus familias, en las regiones de Madrid y Murcia en España. Se realizaron entrevistas en profundidad a miembros de familias migrantes, expertos y expertas en migración, personal de la administración pública, maestros y maestras, y miembros de ONG y asociaciones de migrantes. Las narrativas de los y las jóvenes se recogieron a través de grupos de discusión, entrevistas en profundidad y observación participante. Entre el año 2010 y 2012 se desarrolló el proyecto "Políticas migratorias, transnacionalismo familiar y estratificación cívica. Migraciones latinoamericanas hacia España"1 , con un trabajo de campo etnográfico multisituado (origen y destino) con familias migrantes latinoamericanas, para explorar las formas de reorganización familiar desplegadas por la población migrante latinoamericana en el marco de una nueva crisis económica global y de endurecimiento de las políticas de control migratorio en los países europeos, tomando como uno de los casos de estudio las migraciones de Colombia hacia España. Se realizaron entrevistas en profundidad a expertos, abogados, personal de la administración pública, técnicos/as de inmigración y miembros de ONG y asociaciones migrantes, en el ámbito estatal, autonómico y municipal, en Colombia, Cataluña y Madrid. El estudio se complementó con la recopilación y análisis de fuentes secundarias, documentos (legislaciones, planes, informes), y las declaraciones de representantes institucionales en los medios de comunicación de referencia. Finalmente el artículo se sustenta en los resultados preliminares del proyecto "El sujeto del retorno en el marco transnacional: configuración de subjetividades y trayectorias de inserción en las migraciones internacionales colombianas" ${ }^{2}$, donde se

\footnotetext{
${ }^{1}$ Proyecto (CSO2009-13649 Subprograma SOCI) financiado por el Plan I+D+i del Ministerio de Ciencia e Innovación español. Equipo de investigación: Claudia Pedone (IP), Sandra Gil Araujo, María Margarita Echeverri y Belén Agrela. http://www.ciimu.org/pidpmi/

${ }^{2}$ Investigación llevada a cabo por el Grupo de investigación Géneros y Nuevas Ciudadanías de la Facultad de Psicología de la Pontificia Universidad Javeriana de Bogotá.
} 
han explorado las trayectorias de la migración de retorno de los y las colombianas, a través de 25 entrevistas en profundidad con migrantes retornados, funcionarios encargados de la política migratoria y los programas de retorno en Colombia, resaltando posiciones de género, generación, clase socioeconómica y nivel educativo.

A continuación, en el artículo se hace un breve repaso de la configuración de las migraciones internacionales colombianas, poniendo especial atención en España como país de inmigración. Se destacan los datos que han caracterizado la composición de la población colombiana en el país ibérico. Posteriormente se analizan los tres factores que han incidido de manera importante en el trazado de las migraciones familiares de la población colombiana hacia España. En primer lugar, se muestra como el incremento de la migración femenina a finales de la década del noventa, posicionó la migración familiar al visibilizar a las mujeres, niños, niñas y jóvenes dentro del grupo doméstico como actores decisivos en la migración, en ambos extremos de la cadena migratoria. Se inician en este marco la construcción de estigmatizaciones y representaciones sociales sobre la población joven inscrita en procesos de migración familiar, liderados principalmente por mujeres, que obstaculizaron en muchos casos los proyectos de reagrupación familiar. En segundo lugar, se muestra como la entrada en vigor del visado para la población colombiana en el año 2002 marcó un hito en el trazado de los proyectos migratorios familiares, llevando a las familias a ensayar múltiples estrategias para sortear las políticas y las regulaciones migratorias restrictivas. En tercer lugar, se analiza el impacto de la crisis económica y financiera en los proyectos migratorios y familiares de la población colombiana asentada en España. Se reducen las reagrupaciones familiares, se ensayan estrategias de retorno selectivo y escalonado de algunos integrantes de las familias, se experimentan re-emigraciones, y se abren nuevas rutas migratorias hacía países latinoamericanos y centroamericanos. Por último, en las notas finales se destaca como la precariedad jurídica y laboral de la población migrante, los crecientes obstáculos para la migración familiar en las sociedades de inmigración y la actual crisis financiera y económica mundial están consolidando el transnacionalismo familiar y la dispersión geográfica de los miembros de muchas familias migrantes.

Equipo de investigación: María Margarita Echeverri B. (IP), María Lucia Rapacci, Marcela Rodríguez, Carol Pavajeau. 


\section{EL MOVIMIENTO MIGRATORIO DE LA POBLACIÓN COLOMBIANA HACÍA ESPAÑA}

Colombia ha sido un país migrante por excelencia. Los datos dan cuenta de desplazamientos de todo tipo y por diversas causas: migraciones del campo a la ciudad, desplazamiento interno forzado ${ }^{3}$, refugiados que cruzan a países vecinos como Ecuador ${ }^{4}$, migraciones intrarregionales y migraciones internacionales. La migración internacional de la población colombiana se remonta aproximadamente a la década de los años sesenta del siglo $X X$, coincidiendo con el fin de la denominada época de la violencia (1940-1950) y el inicio de la guerra irregular que se prolonga hasta hoy. Los principales destinos de los colombianos para esta época se trazaron hacia dos puntos importantes: Estados Unidos y Venezuela, donde las reformas a las leyes de inmigración y las redes sociales, políticas y económicas en el primer caso; y la demanda de mano de obra de la economía venezolana por el auge petrolero, se constituyeron en los factores que influyeron de manera determinante en este proceso migratorio (Guarnizo, 2006 y 2008).

Para el período 1965-1975, Venezuela, Estados Unidos, Ecuador y Panamá eran el destino del $90 \%$ de los migrantes colombianos (Cárdenas y Mejía, 2006). Entre los años sesenta y ochenta, Europa se constituía en el principal destino de las elites socioeconómicas, refugiados políticos de izquierda, intelectuales y artistas, y una proporción de colombianos, principalmente mujeres, que en los setenta migró a Inglaterra tras la llamada del gobierno inglés para reclutar mano de obra extranjera no calificada en el sector servicios (Guarnizo, 2008). Hacia mediados de los ochenta otra oleada se da hacia el sur de Estados Unidos. La caída del PIB y el gran incremento del desempleo en Colombia, se sumaron a la ampliación transnacional del narcotráfico,

\footnotetext{
${ }^{3}$ Las cifras del desplazamiento forzado interno reflejan su dimensión. De acuerdo con la Unidad para la Atención y Reparación Integral a las Víctimas (2014) a 1 de mayo de 2014 se registran 6.431.981 victimas en Colombia, de las cuales 5.576 .168 se constituyen por desplazamiento forzado interno. El Observatorio del Desplazamiento Interno -organismo dependiente del Consejo Noruego de Refugiados- aseguró en 2007 que en Colombia había cuatro millones de desplazados internos, es decir, casi uno de cada diez ciudadanos, siendo superado solo por Sudán, con 5,8 millones de desplazados internos (Informe CEAR, 2008).

${ }^{4}$ Según datos de ACNUR, a septiembre de 2013, el Estado ecuatoriano ha reconocido a 54.865 personas refugiadas en el país, donde el $98 \%$ son colombianos. Desde el año 2000 , 170.965 personas han solicitado el reconocimiento de la condición de refugiado en el Ecuador. Cerca del $23 \%$ de ellos son niños, niñas y adolescentes (ACNUR, 2014).
} 
precipitando la salida de muchos colombianos (Guarnizo, 2008; Actis, 2009). A mediados de la década de los noventa, en medio de la intensificación de las violencias -conflicto armado, narcotráfico y delincuencia común- y de la crisis económica de fin de siglo, creció exponencialmente la salida de sectores de la población de clase media y alta. Según datos del Departamento Administrativo de Seguridad (DAS) entre 1996 y 2006 salieron de Colombia aproximadamente 2.1 millones de personas (DANE, 2007).

Actualmente el éxodo colombiano se ha dispersado por todo el mundo, adquiriendo un efecto de carácter global. Según cálculos del Ministerio de Relaciones Exteriores de Colombia, los colombianos se ubican en más de veinte países de cuatro continentes (Cárdenas y Mejía, 2006). Para 2005 se estimaba que había emigrado el $7.5 \%$ del total de la población colombiana y se concentraba en Estados Unidos (34,6\%), España (23\%), Venezuela (20\%), Ecuador (3,1\%), Canadá (2,2\%), Panamá $(1,4 \%)$, México $(1,1 \%)$ y Costa Rica $(1,1 \%)$ (DANE, 2005).

Se estima que la mayoría de los y las colombianas ha salido del país tras la búsqueda de contextos económicos más favorables y seguros, huyendo principalmente de la crisis económica y las altas tasas de desempleo, especialmente durante la crisis de los años noventa (Guarnizo, 2003; Garay y Medina, 2007). Sin embargo, otros factores han jugando un rol determinante, como son los motivos de seguridad relacionados con el conflicto interno, la persecución y la incertidumbre política generadas por la agudización del conflicto armado y las violencias de diverso tipo (Cárdenas y Mejía, 2006; Guarnizo, 2008).

A finales de la década del noventa, la consolidación de España como país de inmigración para la población colombiana estuvo marcada por diversos factores como el acelerado crecimiento económico español, la dinámica del mercado de trabajo, el acceso a servicios sociales, la lengua común, que sumados a la configuración de redes migratorias entre regiones de origen y de destino y una menor dificultad para migrar hacia España (por la exención del visado hasta 2001) en comparación con EE.UU., incidieron en el aumento de la intensidad migratoria (Garay y Medina, 2007). Según la Encuesta Nacional de Inmigrantes (ENI, 2007), el $11 \%$ de los inmigrados colombianos llegó a España antes de 1997, el $67 \%$ lo hizo entre 1997 y 2001, y el 22\% restante llegó a partir de 2002 (Actis, 2009). 
El Censo español del año 2001 contabilizó 160.000 colombianos, en 2002 sumaban 205.000 y en 2005 superaron los 280.000. A partir de entonces su crecimiento fue más moderado, hasta alcanzar 326.000 personas a comienzos de 2008 (Actis, 2009). A partir del año 2002, el ingreso de la población colombiana a España descendió, debido a la entrada en vigor del visado para los colombianos, y al inicio de una política de regulación y ordenación de flujos migratorios a través del Programa Global de Regulación y Coordinación de Extranjería y la Inmigración (Programa GRECO), en el marco del cual se firmó el primer acuerdo bilateral con Colombia el 21 de mayo de 2001 (Sanabria, 2008). Para 2003 fueron 1.931 colombianos quienes llegaron a España a través del programa GRECO, de los cuales 1.531 venían con contrato de temporada (Geronimi, Cachón y Texidó, 2004).

La población colombiana en España se ha caracterizado por ser uno de los colectivos con más alta feminización, si bien la proporción entre hombres y mujeres se ha ido equiparando en el tiempo. Los datos de la ENI (2007) muestran que el $70 \%$ de la población migrante llegada desde Colombia antes de 1997 -y que permanecía en España en el momento de la encuesta- era femenina. Para 2001 las mujeres colombianas representaban el 60\%, un año después descendió al 58\%, para ubicarse en 2007 en el 56,5\% sobre el total del colectivo (Actis, 2009). Igualmente destaca la presencia de niños, niñas y jóvenes. Según la ENI, en 2007 el $39 \%$ de los y las colombianas era menor de veinticinco años, el 53\% tenía entre veinticinco y cuarenta y cuatro años, y el $17 \%$ cuarenta y cinco años o más. Para el segmento infantil y juvenil, los datos muestran que a comienzos de 2008 había unos 35.000 menores de quince años nacidos en Colombia, que se suman al registro de partos, que indica que entre 1998 y 2007 nacieron en España otros 35.000 hijos de madres colombianas (Actis, 2009).

En cuanto a su estatus jurídico, los datos de la ENI 2007 indican que entre enero de 2000 y 2002 alrededor del $70 \%$ de la población colombiana se encontraba en situación irregular, mientras que para el 2008 los migrantes irregulares serían alrededor de 26.000, lo que supone un $14 \%$ del total de empadronados. Por su parte, la población nacionalizada se incrementó entre 2001 y 2008, pasando de 13.000 a 46.000 personas. La gran mayoría provienen del Departamento del Valle (28\%), seguidos por Risaralda (12\%), Antioquia (11\%), y Bogotá (9\%). Las zonas de asentamiento en España son igualmente diversas, donde 
Madrid recibe el $24 \%$ del total de migrantes colombianos, seguida de Cataluña (16\%), Comunidad Valenciana (15\%), Canarias y Andalucía (7\%) (Actis, 2009).

Los datos revelan la existencia de redes migratorias específicas, que vinculan de forma preferente ciertos lugares de origen con determinadas zonas de destino, construidas sobre la base de fuertes redes migratorias familiares transnacionales. Así la ENI en 2007 muestra que el $68 \%$ de los inmigrados desde Colombia tomaron la decisión de viajar por la influencia de un conocido ya radicado en España, que en el $76 \%$ de los casos era un familiar. Únicamente el $13 \%$ reportó haber llegado sin contactos previos (Actis, 2009). Los datos evidencian entonces, que la migración colombiana hacía España es eminentemente familiar y liderada por mujeres. Según el Censo de población de 2011, los colombianos en España sumaban 250.087 personas, de las cuales el $55.3 \%$ eran mujeres (INE, 2013a).

Debido al efecto combinado de la emigración y de la adquisición de la nacionalidad española para el primer semestre de 2013 la población extranjera se redujo en 202.193 (un 4,0\%). A 1 de enero de 2013 los colombianos contabilizaban 223.140 personas, mientras en julio de 2013 eran 194.812 (INE, 2013b). A pesar de la crisis económica actual, los colombianos siguen ocupando la cuarta posición dentro de las nacionalidades de extranjeros más numerosas en España. En 2013 las mujeres representan el $58 \%$ de la población colombiana residente en ese país ${ }^{5}$. (INE, 2013a).

\section{LA FEMINIZACIÓN DE LAS MIGRACIONES Y LAS FORMAS DE ORGANIZACIÓN FAMILIAR DE LA POBLACIÓN COLOMBIANA EN ESPAÑA}

El inicio del Siglo XXI marcó un punto de inflexión en relación a la intensidad, aceleración y feminización del flujo migratorio colombiano hacia España, que en el año 2001 estaba compuesto en un 60\% por mujeres (Actis, 2009). El trabajo de campo durante más de una década con familias colombianas migrantes en España evidencia que la presencia femenina en estas corrientes migratorias se explica por

\footnotetext{
${ }^{5}$ El incremento de la proporción de mujeres en la migración colombiana podría estar indicando un mayor retorno de varones, vinculado con el mayor desempleo masculino entre los migrantes en los primeros años de la crisis.
} 
diferentes factores que se conjugaron para aquella época. Por una parte, en América Latina, la privatización de servicios como la salud o la educación, y el recorte del gasto público transfirieron la obligación de cubrir estas necesidades a las familias, presionando a las mujeres a buscar vías de supervivencia, mediante su inserción en el mercado laboral dentro de los segmentos precarios e informales, en paralelo a su rol de jefas de hogares monoparentales (Gil Araujo, 2005). Por otra parte, la demanda de mano de obra femenina para el servicio doméstico y de cuidados, por parte de los países del norte (Gregorio y Ramírez, 2000; Parella, 2003; Pedone y Gil Araujo, 2008; Gil Araujo y González 2014), impulsaron a las mujeres colombianas a iniciar el viaje a España como primeros eslabones de las cadenas migratorias. Así, para el $48 \%$ de las migrantes colombianas la emigración a España supuso un primer empleo en el servicio doméstico, que casi no se realizaba en Colombia (6\%) (Sanabria, 2008).

\begin{abstract}
Primero llegué yo porque para uno es más fácil conseguir trabajo. Y empecé limpiando como todo el mundo y así estuve como casi dos años. Ya él [marido] llegó, estuvo trabajando ahí en la guardería de jardinero, que fueron los que le dieron los papeles [...] Yo en Colombia no trabajaba. Soy graduada en educación preescolar, y nunca trabajé, me dediqué fue a mi hijo. Es más yo allá nunca hacia nada, acá fue donde me tocó venir a limpiar, pero bueno por algo se empieza (Entrevista a Piedad, mujer colombiana que migró a España en 1998 y reagrupó a su hijo y a su cónyuge en 2000. Madrid, 2001).
\end{abstract}

Fueron estas mujeres los primeros eslabones de las cadenas migratorias (Pedone, 2006), quienes iniciaron procesos de migración y de reagrupación por fuera de los canales establecidos por el Estado (de cónyuges, hijos e hijas, padres y madres, hermanas, entre otros) desde la década del noventa, aprovechando la exención de visado que estaría vigente hasta finales de 2001. Sin embargo, tras el anuncio de la obligatoriedad del visado a partir del año 2002, la reagrupación de sus cónyuges y especialmente de sus hijos se aceleraron. En pocas semanas se planearon y llevaron a cabo procesos de reagrupación familiar autónomos -ingresando a España como turistas-, a pesar de la situación jurídica irregular, el poco tiempo de residencia y la precariedad 
laboral, en tanto más adelante se tornaría más complicado cruzar la frontera (Echeverri, Pedone y Gil Araujo, 2013).

A partir de entonces, la reagrupación familiar y los proyectos migratorios familiares liderados por mujeres se convirtieron en la vía de migración que prevalece hasta hoy, incrementándose la presencia de niños, niñas y jóvenes migrantes ${ }^{7}$, y aumentando la tendencia hacia la equiparación entre sexos dentro del colectivo debido a la reagrupación de sus cónyuges, sin reportarse hasta la actualidad un equilibrio total entre hombres y mujeres.

Al igual que ocurrió con la migración ecuatoriana (Pedone, 2006), este proceso de feminización dio un giro a los proyectos migratorios familiares de la población colombiana, y visibilizó a las mujeres, niños, niñas y jóvenes dentro del grupo doméstico como actores decisivos en el juego de las relaciones de poder, al romper con una representación social muy afianzada, en relación a la organización y ejecución de los proyectos migratorios internacionales como una decisión eminentemente masculina. Nuestra investigación mostró la manera en que los proyectos migratorios familiares liderados por las mujeres generaron reconfiguraciones en las relaciones de género $y$ generacionales; provocando una hipervisibilización y estigmatización de los hijos e hijas de familias migrantes colombianas a ambos lados del Atlántico (Carrasquilla y Echeverri, 2003; Echeverri, 2005 y 2010), basadas en el cuestionamiento del ejercicio de la maternidad transnacional de las mujeres migrantes.

Se instaló así, un discurso social, académico y político que fue configurando a las familias migrantes como problema social tanto en origen como en destino. En Colombia se afirmaba que la salida de las mujeres provoca la "desintegración familiar" y trae "disfuncionalidades" (embarazo adolescente, bajo rendimiento escolar y violencia de pandillas juveniles) a las familias migrantes por el "abandono" de los hijos e hijas de estas mujeres. Simultáneamente, en España, nuestras

\footnotetext{
${ }^{6}$ Similares caracteristicas para los procesos migratorios de la población colombiana hacía España han sido analizados por otras investigaciones (UN-INSTRAW/OIM, 2007; Sanabria, 2008).

${ }^{7}$ Colombia es uno de los países que más jóvenes aporta a la inmigración en España. Para 2004 eran 82.939 los colombianos entre quince y veintinueve años empadronados, que representan el $10 \%$ del total de jóvenes extranjeros y $34 \%$ de los colombianos empadronados (Cachón, 2005).
} 
investigaciones revelaban que los y las jóvenes colombianos involucrados en procesos de migración familiar, empezaron a cargar la estigmatización del discurso institucional y popular, que inscribe su "inintegración" y bajo rendimiento escolar en el hecho de ser hijos e hijas de la migración femenina. En el año 2003 se disparan las alarmas, asociando de manera generalizada a la población joven de origen latinoamericano con la aparición de las mal llamadas "bandas latinas", atribuyéndole su participación en estos grupos al abandono de sus madres que, según algunos discursos, son las culpables de estos hechos por sus largas jornadas laborales (Echeverri, 2010a y 2005).

Dichas estigmatizaciones sobre la población joven inscrita en procesos de migración familiar liderados principalmente por mujeres, dieron un giro al trazado de los proyectos migratorios familiares, viéndose truncados algunos procesos de reagrupación de los hijos y las hijas que en origen esperaban unirse al grupo familiar, y en otros casos iniciando procesos de retorno de las generaciones jóvenes. Es el caso de Ángela, una madre colombiana que migró en 1999 con su hijo mayor a Murcia, esperando reagrupar a sus dos hijos menores tan pronto se hubiese instalado en España. Pasados los años decide no reagruparlos en tanto las representaciones e imágenes de la migración juvenil reflejaban a unos jóvenes que en territorio español "se perdian". Los hijos de Ángela que permanecian en origen empezaban a tener voz y voto en el trazado de sus proyectos migratorios, eligiendo culminar su educación en Colombia.

Lo he pensado mucho, sí, pero tampoco he hecho mucho empeño en traerlos porque he visto muchos espejos aqui de los niños que han traído. Porque los niños llegan aquí, y de repente aquí no quieren estudiar, de repente aquí, como es un cambio tan brusco de colegio, de compañeros, de costumbres, aquí yo los veo que ya no quieren estudiar. Entonces pues eso también no me ha dado a mi mucho impulso para traerlos [...] Y ellos también [allí] lo del estudio lo escuchan mucho, niños que han venido aquí y han ido allí de paseo, o se han vuelto porque se han aburrido, [y les dicen] que el estudio aquí es muy diferente al de allá [...] Es otra cosa que a ellos también les ataja un poco [...] Entonces esas son cosas que yo escucho mucho y no, no, no quiero como vivirlas con mis hijos aquí, no quiero (Entrevista a Ángela, Murcia, 2005). 
En esta línea, los discursos sociales, políticos e institucionales estigmatizantes y culpabilizadores sobre las mujeres migrantes colombianas, generaron reajustes en las formas de organización de sus familias, y se iniciaron infinidad de negociaciones y conflictos, que debian sortear desde la ausencia/presencia los niños, niñas y jóvenes, quienes reconfiguraron sus sentidos de pertenencia, sus relaciones y vínculos afectivos, tanto en origen como en destino. Las mujeres colombianas empiezan a privilegiar la configuración de vínculos familiares transnacionales, incluido el retorno de algunos jóvenes que habian migrado con sus familias a España.

Así, las ideologías de género permiten identificar importantes diferencias a la hora de abordar la separación de los hijos e hijas dentro de los procesos migratorios, según se trate de los padres (práctica normalizada que no pone en crisis las relaciones paterno-filiales), o las madres, por lo general culpabilizadas por la ruptura familiar, estigmatizadas y desaprobadas socialmente (Parreñas, 2001; Solé, Parella y Cavalcanti, 2007). Estigmas y narrativas que incidieron en la configuración de familias transnacionales para el caso de la migración colombiana.

A partir del año 2002 se suman otros factores que dificultan o impiden la reagrupación de las familias migrantes y abocan nuevas estrategias en la que todos sus miembros están involucrados. Los controles fronterizos aumentan y las estrictas políticas de inmigración contribuyen activamente a la formación de familias transnacionales (Sorensen y Guarnizo 2007).

\section{FRONTERAS POLÍTICAS, BARRERAS JURÍDICAS: LAS REAGRUPACIONES FAMILIARES COLOMBIANAS ANTES Y DESPUÉS DE LA IMPOSICIÓN DEL VISADO A ESPAÑA}

El control y regulación de los flujos migratorios en España que comprende los sistemas selectivos de acceso al territorio, al mercado de trabajo y las restricciones a las políticas de reagrupación familiar, han limitado de manera significativa la migración familiar, cercenando el derecho a vivir en familia, e incidiendo en las formas de organización familiar. En este marco de restricción y cierre de fronteras, el año 2002 marcó un hito en los proyectos migratorios y las formas de organización de las familias migrantes colombianas, tras la imposición del visado de 
ingreso a España. A continuación se analizarán las diversas estrategias impulsadas por las familias migrantes, que ponen de manifiesto cómo las políticas y regulaciones migratorias han reconducido, obstaculizado y resignificado los proyectos migratorios familiares de esta población.

Con la entrada en vigor del visado para el año 2002 se cerró la posibilidad de llevar a cabo procesos de reagrupación familiar informales de los cónyuges, hijos e hijas como venía sucediendo, sumado a que un alto porcentaje de la población colombiana para aquella época se encontraba en situación jurídica irregular, en tanto habían ingresado a España como turistas. Así, principalmente a las mujeres se les dificultaba cada vez más cumplir con los requerimientos jurídicos y económicos para la reagrupación de sus hijos e hijas, en consonancia con las escasas vías de migración regular, su permanencia en el servicio doméstico, los obstáculos en el acceso a la vivienda y la precariedad e informalidad del mercado de trabajo al que por lo general pueden acceder (Gil Araujo y González, 2014).

En nuestro trabajo de campo relevamos relatos que dan cuenta del inicio de los tortuosos y largos procesos de regularización jurídica que emprendieron los padres y especialmente las madres colombianas, y que impusieron grandes dilaciones temporales en la reagrupación de las hijas e hijos llevadas a cabo con posterioridad al año 2002. Es el caso de Gloria, quien llega a España el veinticuatro de diciembre de 2001, aprovechando que podía ingresar sin visado. Al salir tan de repente decide dejar a su hija de trece años en Colombia porque no sabía con qué se iba a encontrar. En 2002 sin forma de regularizar su situación, la familia de la anciana que cuidaba le ayuda para reagrupar a su hija Marcela a través de una visa de estudiante, pero le negaron la visa. Su siguiente estrategia fue casarse y encontró con quien hacerlo. El hijo de una de las ancianas que seguía cuidando se ofreció en 2004 a casarse con ella, pero se arrepintió en las puertas del juzgado. En 2005 logró acogerse al proceso de regularización, donde obtuvo los papeles. Solo bastaba esperar otro año para poder reagrupar a su hija, en tanto la normativa para la reagrupación familiar exigía la tarjeta de residencia renovada. En febrero de 2006 con el comprobante de renovación tramitó la reagrupación de Marcela, que después de diversas trabas que logró superar, se aprobó por fin diecinueve días antes de que la joven cumpliera dieciocho años. "Nos salvamos por un pelo" (Entrevista Gloria, Murcia, 2007). 
Los requisitos que debían cumplir los reagrupantes en relación al tipo de permisos, ingresos económicos, tipo de vivienda, hicieron que las políticas de reagrupación familiar españolas tuvieran el efecto de determinar el perfil y la edad de los reagrupados. Así, algunas familias colombianas empezaron a organizar los procesos de reagrupación de sus hijos de manera escalonada, donde se privilegiaba en algunos casos a los hijos e hijas que estaban próximos a cumplir dieciocho años, edad límite establecida por la normativa. En otros casos se reagrupaban primero a los más pequeños, en tanto se manifestaba en ellos una tristeza profunda que sus hermanos mayores podían resistir, hasta que sus padres o madres tuvieran la condición exigida por la normativa para poder reagruparlos. Es el caso de Estefania, quien nos contó en Madrid:

Yo no sé qué pasó, yo estaba muy chiquita, pero algo así que el banco se fue a la quiebra, nos embargaron la casa, tuvimos que vender todo, tuvimos que irnos a vivir a donde mi abuela. Luego a mi papá le propusieron venirse pa`[sic] España, y acá a él le dio muy duro, estuvo como un año, se vino solo y acá trabajó en ferias, trabajó en un poco de sitios, en el mercadillo. Pero muy mal estaba, muy duro, luego tenía depresión. Luego se vino mi madre, y como al poco tiempo, creo que a los dos años, se tuvo que venir mi hermano, porque a mi hermano le estaba dando una depresión allá en Colombia, una depresión de no verlos, y entonces él estaba muy chiquito y se lo tuvieron que traer. Y yo como soy la más fuerte, así de los dos, entonces yo sí aguanté más tiempo y cuando estuvieron más establecidos y todo, entonces ya llegaron, ya mandaron por mí y ya, aquí llegué (Estefania, joven colombiana que migró a Madrid en 2003. GD8 jóvenes colombianos, Madrid, 2007).

Para los hijos e hijas que ya habían cumplido la mayoría de edad se inicia el ensayo de diversas estrategias para reagruparlos, privilegiando procesos autónomos por la vía de los contingentes de trabajo, en tanto en 2002 se suspendieron las tramitaciones de permiso de trabajo por la Vía General. Es el caso de Miriam, quien migró a España en 2001. Su intención era reagrupar a sus hijos, para lo cual puso en marcha distintas estrategias. Para reagrupar al hijo menor, tuvo que esperar siete años mientras logró obtener los papeles y cumplir con todos los requisitos exigidos por la normativa para la reagrupación de un menor de edad. Para reagrupar a una de sus hijas mayores, recurrió a la vía de los contingentes de trabajo, que según Miriam fue mucho más facil que llevar a su hijo menor a España. 
A ella para poderla traer, tocaba con contrato de trabajo, porque como ella es mayor, no la podía hacer por reagrupación [...] Entonces yo para traer a mi hijo, al principio, primero tenía que conseguir un piso adecuado, porque eso van del ayuntamiento, no sé que, vigilando y todo. Entonces a ella era más fácil traerla [...] pues porque era con un contrato de trabajo. A mi hijo tocaba la reagrupación, tocaba un montón de papeles, y aparte tener un piso [...] A él era mucho más complicado por ser joven (Entrevista a Miriam, Madrid, 2010).

En esta línea, nuestras investigaciones (Echeverri, 2005 y 2010) muestran cómo las variaciones en las modalidades de reagrupación familiar escalonadas y con grandes distancias espaciales y temporales que se sucedieron a partir del año 2002, influyeron en las trayectorias identitarias y en los procesos de incorporación en la sociedad de inmigración de los hijos e hijas de las familias migrantes colombianas. Algunos de los niños, niñas y jóvenes que habían llegado a España por reagrupación antes de la imposición del visado, relataron que sus familias habían migrado al completo con periodos relativamente cortos de separación. Sus relatos no narraban tanto malestar, al contrario, su migración se circunscribía en el éxito y bienestar que sentían por reencontrarse con su familia, a pesar de la situación de irregularidad que atravesaban todos pasados los primeros meses de estancia.

Los y las jóvenes que llegaron desde Colombia por reagrupación familiar posterior al año 2002 comentaban la dispersión de sus familias y las reagrupaciones escalonadas con largos tiempos de espera. Estos jóvenes experimentaron procesos de inserción muy difíciles y dolorosos, donde el reencuentro con sus familias se convirtió en un conflicto añadido a sus trayectorias en España. Al interior de las familias se sucedieron infinidad de negociaciones afectivas y emocionales, donde el sentimiento de culpa de las madres migrantes se constituia en un eje fundamental en ambos extremos de la cadena migratoria.

En Colombia se reforzaron los discursos sociales y políticos que señalaban los procesos migratorios familiares liderados por mujeres como el origen de la desintegración familiar por el abandono de los hijos e hijas de las familias migrantes ${ }^{8}$, sin tener en cuenta que cada vez se

\footnotetext{
${ }^{8}$ Para un análisis detallado sobre las formas de concebir las relaciones entre migración, familia y género en el discurso político y en las políticas públicas colombianas ver Echeverri, Pedone y Gil Araujo, 2013.
} 
incluian más restricciones a la política migratoria familiar en España, que hacian casi imposible para estas mujeres reagrupar a sus hijos e hijas en cortos periodos de tiempo. Así, por ejemplo, en 2009 la Procuraduría General publicó un informe sobre los hijos de la migración titulado "Hijos huérfanos de padres vivos", que recibió una dura crítica de algunos sectores académicos. Por su parte los lineamientos de la Política Integral Migratoria en Colombia (Documento Conpes 3603, 2009), reflejan la "natural" conexión entre migración femenina y disfuncionalidades familiares. Se afirma en el documento que: "El denominado fenómeno de la feminización de las migraciones y su impacto en la familia, es un tema que merece especial atención", destacando una clara preocupación por los "impactos en las mujeres que se quedan y sus hijos" y sobre todo por los "cambios en el modelo de familia". En la misma sintonía una experta en migraciones en Colombia sostenía: "Aquí ha sucedido eso, lo que hemos analizado, donde están los mayores sitios de migración, donde se han ido más cantidad de personas, por lo menos aquí en este municipio, es donde hay más focos de violencia de los jóvenes" (Entrevista a experta en migración, Risaralda, Colombia, 2010).

Actualmente, la política migratoria en España exige cada vez más requisitos que prolongan en un "sin fin" el proceso, lo que supone periodos cada vez más largos para que los hijos e hijas se puedan encontrar con sus padres y madres en alguno de los dos lados del Atlántico. Últimamente a las restricciones impuestas por el visado de ingreso a los colombianos a España y por la política de reagrupación familiar, se van sumado prácticas informales que restringen y disminuyen el porcentaje de resoluciones favorables a las solicitudes de reunificación familiar, como el pedido de informes que no se desprenden del reglamento sino que dependen del funcionario de turno, o en otros casos el vencimiento de los términos de algunos documentos frente a los largos periodos que transcurren para expedir las resoluciones (Notas del trabajo de campo Colombia, 2010).

Nuestras indagaciones han mostrado como las estrategias selectivas y escalonadas para reagrupar a los hijos e hijas impactaron en las relaciones de género y generacionales al interior de las familias, donde las mujeres, los niños, niñas y jóvenes son actores importantes de estas

\footnotetext{
${ }^{9}$ El Tiempo, 12 de enero de 2009.
} 
dolorosas tramas que la política de reagrupación familiar y el cierre de las fronteras están imponiendo.

\section{LA CRISIS ECONÓMICA Y LOS PROCESOS DE RETORNO Y RE- EMIGRACIÓN DE LAS FAMILIAS COLOMBIANAS EN ESPAÑA}

El año 2009 marca un nuevo hito en la configuración de los proyectos migratorios familiares de la población colombiana asentada en España. El ciclo económico expansivo en el país ibérico, que se vivía para finales de la década del noventa, concluyó de manera drástica, afectando principalmente a la agricultura y la construcción, sectores de la economía donde se concentra gran parte de la población migrante, especialmente los varones (Colectivo loé, 2011b). En el marco de la crisis económica, hasta la primera mitad de 2013 el número de ocupados descendió dramáticamente (-3,7 millones de empleos), afectando principalmente a los hombres inmigrados (de 79,7\% en 2007 a 53,6\% en la primera mitad de 2013), y a las mujeres inmigradas (de 60,7\% a 48,2\%) (Colectivo loé, 2013).

Según los datos, los hombres migrantes han padecido más que las mujeres la pérdida de empleo, quienes han incrementado su presencia en el mercado laboral ante la disminución de los ingresos experimentados por muchos hogares migrantes debido a la pérdida de empleo de los varones (Colectivo loé, 2012), con impactos sobre la vida familiar y las relaciones de género; en tanto no se registran redistribuciones de las tareas domésticas, por lo que cabe suponer un incremento de las cargas para la población femenina. Sin embargo, el empleo femenino que se vio menos afectado al comienzo de la crisis, empieza a caer de forma notable desde 2012, acercándose a los niveles de retroceso de la población masculina (Colectivo loé, 2013). La tasa de desempleo en el caso de la migración colombiana pasó de 11,3\% en 2005 a $31.2 \%$ en 2011 , con una tasa similar entre hombres y mujeres, donde los hogares de migrantes colombianos con todos sus integrantes en situación de desempleo pasaron de representar el 5,6\% en 2007 al 13,1\% en 2011 (Colectivo loé, 2012).

La crisis económica y el desempleo han afectado sustancialmente a la población joven. Se registran fuertes caídas de la tasa de actividad en los menores de 25 años tanto para los migrantes (de 61,7\% a 48,6\%) como para la población autóctona (de 50,6\% a 39,3\%), mostrando una 
retracción de la actividad de los jóvenes y las mujeres migrantes. En esta línea, se registra una notable caída de las remesas enviadas a los países de origen, que entre 2007 y 2012 disminuyeron un 23\%, y sugiere un empeoramiento creciente de los ingresos monetarios de la población inmigrada (Colectivo IOE, 2013).

La agudización de la crisis económica y financiera en España viene acompañada por un endurecimiento de la política de migración familiar y el establecimiento de nuevas restricciones en los procesos de reagrupación, como los incluidos en Ley Orgánica 2/2009, de 11 de diciembre, de reforma de la Ley Orgánica 4/2000, de 11 de enero, sobre derechos y libertades de los extranjeros en España y su integración social, donde se establece que sólo los inmigrantes con permisos de larga duración podrán reunificar a los ascendientes del reagrupante y de su cónyuge, en línea directa y en primer grado, cuando estén a su cargo, sean mayores de sesenta y cinco años y existan razones que justifiquen la necesidad de autorizar su residencia en España (Boletín Oficial del Estado, 2009: 104989).

En este marco, los procesos de reagrupación familiar se han reducido de manera drástica y las familias migrantes colombianas transforman sus estrategias, que incluyen el retorno selectivo y escalonado de sus miembros a Colombia, las re-emigraciones, sobre todo de la población joven, y el desarrollo de nuevas rutas migratorias hacía países latinoamericanos y centroamericanos.

De esta manera, en nuestro trabajo de campo realizado entre 2010 y 2013 constatamos que los encuentros de las familias migrantes colombianas en España, basados principalmente en el esfuerzo de las mujeres por reagrupar a sus hijos, superando los obstáculos que ha interpuesto la política migratoria, se rompen de nuevo. El endurecimiento de las políticas y la actual crisis económica están generando nuevos procesos de des-agrupación familiar (Juliano, 2012), intensificando los procesos de maternidad y paternidad transnacional.

Las estrategias de retorno y re-emigración están marcadas por el género y la generación, y de manera contundente por el estatus jurídico que hayan obtenido en sus trayectorias los miembros de las familias colombianas en España. Así, hemos encontrado que la mejor posición de las mujeres en el actual contexto de crisis económica, las ha llevado a permanecer en España reafirmando su papel como cabeza de las 
unidades domésticas y su compromiso en las tareas de reproducción y sostenimiento de sus hogares allí donde se encuentren. Según Mejía (2010) en el retorno de la población colombiana se sugiere una menor propensión de las mujeres al retorno o por lo menos, mayor demora en el mismo.

Por su parte, los jóvenes de estas familias empiezan a ensayar nuevas migraciones, tanto al país de origen como a otros lugares, preferentemente el Reino Unido, Francia y Alemania, y recientemente a otros países de América Latina, que fortalecen nuevas rutas de migración para la población colombiana, como son Argentina, Chile, Ecuador, Brasil, entre otros. Estas decisiones están condicionadas por el estatus jurídico de los jóvenes, que en algunos casos deciden los nuevos destinos en el marco de la obtención de la nacionalidad española. Como señala Pajares (2010) la migración de retorno está influenciada por las condiciones en los contextos de destino y de origen, pero también por la posibilidad de volver a migrar. Es el caso de Samuel quien llega a España de catorce años en 1997 con su madre y su hermano. Tras quince años, al obtener su nacionalidad española, decide migrar a Inglaterra en busca de oportunidades laborales que ya no encuentra en España. Su madre sigue en Madrid, donde asegura que a pesar de la situación de crisis y de los recientes recortes en el gasto social, aun puede acceder a los servicios de salud que en Colombia no tendría, frente a un eventual retorno.

A mi me gusta mucho vivir en Madrid, pero necesito trabajar y aquí cada vez está más difícil. ¡i¡Ya me salió la nacionalidad, después de 15 años!!! Me voy a Inglaterra a buscarme la vida y a aprender bien inglés, sino me regreso a Madrid (Entrevista a Samuel, Madrid, 2012).

$\mathrm{Si}$ bien algunos retornos de las familias colombianas han sido encabezados por los hijos, el trabajo de campo desarrollado en 2013 en Colombia nos ha revelado que algunos de estos jóvenes ensayan su retorno y ante el adverso contexto colombiano deciden emigrar de nuevo. Es el caso de Hernán, joven colombiano que llega a España en 1998 con su padre exiliado. En 2013 decide retornar a Colombia, y tras cinco meses de vivir en Bogotá decide regresar a España.

Yo decidi venir a ensayar, yo quería establecerme otra vez aquí en Colombia. Pero en diez días me vuelvo a ir. Yo aquí no me siento seguro, allí se vive más tranquilo, a pesar de lo difícil que está por la crisis. Cuando llegué aquí [a Colombia] 
tuve que hacer mil vueltas y demostrar que yo existía. Las vueltas de la EPS $^{10}$ son un martirio, no tenemos historia crediticia aquí en Colombia y por eso no existimos (Entrevista Hernán, Bogotá, 2013).

En resumen, contrario a lo postulado por algunos discursos políticos, amplificados por los medios de comunicación, sobre el retorno masivo de migrantes ${ }^{11}$, nuestras investigaciones indican que el retorno está siendo selectivo y organizado sobre la base de nuevas estrategias migratorias que se están diseñando y poniendo en práctica, incluidas nuevas migraciones a otros destinos. Los procesos migratorios para las familias colombianas en España, en el marco de la crisis económica actual y las sucesivas restricciones a la política migratoria, se caracterizan por una dinámica escalonada de retornos y reemigraciones que inciden en la organización de estas familias de manera diferenciada en relación al género y la generación. Así, las políticas migratorias y los contextos económicos, políticos y sociales de las sociedades de inmigración y de emigración están afectando las trayectorias de las familias migrantes colombianas, que retoman el ejercicio de la maternidad y la paternidad transnacional, para garantizar la subsistencia de sus integrantes.

\section{NOTAS FINALES}

El Para inicios del siglo XXI, la migración de las mujeres colombianas hacia España visibilizó los complejos proyectos migratorios familiares que se han sucedido en las últimas décadas, sacando a la luz la

\footnotetext{
${ }^{10}$ Entidades Promotoras de Salud, cuya función es organizar y garantizar, la prestación de los servicios de salud que se encuentran en el Plan Obligatorio de Salud y son las responsables de la gestión de riesgos derivados de la enfermedad general o no ocupacional.

${ }^{11}$ Aunque la crisis en España ha supuesto una disminución del saldo migratorio, este se debe más a una reducción de las entradas, que a un incremento de las salidas (193.000 en 2007 y 280.000 en 2009), lo que lleva a pensar que no se presenta un espectacular "éxodo" de inmigrantes (Colectivo IOE, 2011b). Las cifras de empadronados muestran que el volumen de la población colombiana en España siguió creciendo hasta comienzos de 2012, descendiendo solo hasta 2013 (Colectivo loé, 2013). Para el caso español el PREVIE (Programa de Retorno Voluntario de Inmigrantes desde España), reportó 662 colombianos acogidos al programa desde 2003 hasta marzo de 2009 (Pajares, 2010), mientras que el programa español de retorno voluntario para inmigrantes con abono acumulado y anticipado de la prestación por desempleo, un año después de estar en funcionamiento se habían acogido menos de 1600 colombianos (EFE, 2009).
} 
participación de las generaciones jóvenes en el trazado de las múltiples estrategias que estas familias implementan para cruzar las fronteras físicas y simbólicas que se imponen en sus tránsitos migratorios. El papel de las mujeres colombianas como pioneras de la migración hacia España ha generado discursos estigmatizadores que vinculan la emigración femenina al abandono de los hijos, a la desintegración familiar y a otras supuestas "disfuncionalidades" de las familias transnacionales, en ambos extremos de la cadena migratoria. Por su parte, hijos e hijas de esta migración se fueron configurando en el discurso político de los contextos de emigración y de inmigración como sujetos con comportamientos "desviados" y "delictivos".

Los discursos estigmatizantes, que han condenado a los hijos y a las madres colombianas por sus procesos migratorios están asociados a la naturalización que (en origen y destino) sigue perpetuando el papel de las mujeres como reproductoras y cuidadoras, y de los hombres como proveedores, en el seno de familias que "deben ser nucleares" (padremadre-hijos menores) y materializadas en un espacio nacional común y compartido. La circulación de esta narrativa, se constituyó en la primera barrera para llevar a cabo procesos de reagrupación familiar al tiempo que invisibilizó el fundamental aporte de estas mujeres al bienestar, no solo de sus familias, sino también de sus países de origen, como proveedoras de recursos, y principales organizadoras del cuidado transnacional.

A esta dificultad para la vida familiar, en el año 2002 se sumó la imposición del visado para el ingreso de la población colombiana, que evidencia de manera rotunda los perversos efectos que el cierre de fronteras y las restricciones de las políticas migratorias han tenido en las formas de organización familiar de la población migrante. Las políticas de migración familiar están operando como instrumentos de control, restricción y selección de inmigrantes, cercenando el derecho a vivir en familia. Las dificultades para concretar los procesos de reagrupación familiar han promovido la extensión y consolidación de las vínculos familiares transnacionales y los procesos escalonados de reagrupación familiar, con distanciamientos geográficos y temporales prolongados que impactaron negativamente en los reencuentros de madres, padres e hijos y reforzaron la imagen de las mujeres como abandonadoras de su prole, tanto en Colombia como en España. 
La actual crisis económica y financiera en España, acompañada de más restricciones a la migración familiar, está dando lugar a procesos de reorganización de las familias migrantes colombianas. Actualmente al interior de los grupos domésticos se negocia cuál es el miembro que reúne las mejores condiciones para el retorno o la re-emigración, mientras algunos integrantes, por lo general las mujeres, permanecen en España generando recursos económicos para la subsistencia de las familias transnacionales. Las condiciones de origen y destino, producen procesos de des-agrupación familiar, debido a que el retorno es selectivo y escalonado, y liderado en muchos casos por los miembros más jóvenes, quienes diseñan sus estrategias condicionadas por su estatuto jurídico y por las condiciones socioeconómicas de origen y destino, que en algunas ocasiones los llevan a emprender la emigración hacía otros lugares del mundo, participando en el trazado de nuevas rutas para la migración colombiana.

Las experiencias de la migración colombiana que hemos relevado en España y Colombia a lo largo de los últimos diez años confirman que los procesos migratorios se configuran de manera diferenciada en relación al género y la generación y están influidos por las políticas de Estado y los contextos económicos, políticos y sociales de las sociedades de origen y destino(s) de los migrantes. La precariedad jurídica y laboral de la población migrante junto con los crecientes obstáculos para la migración familiar en las sociedades de inmigración alientan la consolidación de formas de organización familiar transnacionales. La dispersión geográfica de los miembros de las familias migrantes parece destinada a convertirse en uno de los rasgos distintivos de las migraciones Sur-Norte del siglo XXI.

\section{BIBLIOGRAFÍA}

ACNUR, 2014, EI ACNUR en Ecuador, http://www.acnur.org/t3/dondetrabaja/america/ecuador/.

Actis, W., 2009, "La migración colombiana en España: ¿salvados o entrampados?", en Revista de Indias Vol. LXIX, n 245 , pp.145 170.

Cachón, L., 2005, “Inmigrantes jóvenes en España”, en A. López, L. Cachón y D. Comas, Informe Juventud en España 2004, Instituto de la Juventud, Ministerio de Trabajo y Asuntos Sociales, Madrid, pp. 697-799. 
Cárdenas, M. y Mejía, C., 2006, "Migraciones internacionales en Colombia: ¿qué sabemos?", en Documento de Trabajo, n 30.

Carrasquilla, M. C. y Echeverri, M. M., 2003, "Los procesos de integración social de los jóvenes ecuatorianos y colombianos en España: un juego identitario en los proyectos migratorios", en Estudios de Juventud n 60 , pp. 89-100.

Castles, S. y Miller, M., 2007, La Era de la Migración: Movimientos internacionales de población, Universidad Autónoma Metropolitana, México DF.

Colectivo loé, 2013, "La población inmigrada ante la crisis: ¿mirando hacia otro lado?", en Boletín Ecos, $n^{\circ} 24$ Septiembre-Noviembre www.fuhem.es/ecosocial, pp. 1-10.

Colectivo loé, 2012, Impactos de la crisis en la población inmigrante, Organización Internacional para las Migraciones, Madrid.

Colectivo loé, 2011b, "Notas sobre los efectos socioeconómicos de la crisis para la población inmigrada", en Papeles de relaciones ecosociales y cambio global, $n^{\circ} 113$, pp. 85-95.

Colectivo loé, 2011a, "Efectos sociales de la crisis. Una evaluación a partir del Barómetro social de España", en Papeles de relaciones ecosociales y cambio global, $n^{\circ} 113, \mathrm{pp} .177-188$.

Comisión Española de Ayuda al Refugiado (CEAR), 2008, La situación de los refugiados en España, informe 2008, CEAR, Madrid.

Consejo Nacional de Política Económica y Social, Departamento Nacional de Planeación, 2009, Documento Conpes 3603, Política Integral Migratoria, Bogotá.

Departamento Administrativo Nacional de Estadística (DANE), 2005, "Jefatura de hogar" Censo General. www.dane.gov.

Departamento Administrativo Nacional de Estadística (DANE), 2007, Proyecciones nacionales y departamentales de población, 2006-2020. http://www.dane.gov.co/files/investigaciones/poblacion/proyepo bla06_20/7Proyecciones_poblacion.pdf.

Echeverri, M. M., Pedone, C. y Gil Araujo, S., 2013, "'Entre la estigmatización y la restricción'. Políticas migratorias y discursos políticos sobre familia, migración, género y generación en países de inmigración y emigración: España y Colombia", en Revista Palobra. Palabra que obra, $\mathrm{n}^{\circ}$ 13, pp. 84-107.

Echeverri, M. M., 2010, "Son diez horas de viaje y cinco años que te meten encima". Proyectos, identidades y vínculos transnacionales de los y 
las jóvenes colombianas en España, Tesis doctoral, Facultad de Ciencias Políticas y Sociología, Universidad Complutense de Madrid, Madrid.

Echeverri, M. M., 2010a, "'Son diez horas de viaje y cinco años que te meten encima'". De lo colombiano a lo latinoamericano: identidades migratorias juveniles en España", en Grupo Interdisciplinario de Investigador@s migrantes (Coord.), Familias, niños, niñas y jóvenes migrantes. Rompiendo estereotipos, IEPALA Editorial, Madrid, pp. 159-172.

Echeverri, M. M., 2005, “Fracturas identitarias: migración e integración social de los jóvenes colombianos en España", en Migraciones Internacionales, 3 (1), pp. 141-164.

Escrivá, A. y Ribas Mateo, N., 2004, Migración y Desarrollo. Estudios sobre remesas y otras prácticas transnacionales, Consejo Superior de Investigaciones Científicas e Instituto de Estudios Sociales de Andalucía, Córdoba.

Garay, L. J. y Medina, M.C., 2007, La migración colombiana a España. El capítulo más reciente de una historia compartida. Ministerio de Trabajo e Inmigración, Observatorio Permanente de Inmigración, Madrid.

García Borrego, I., 2008, Herederos de la condición inmigrante: adolescentes y jóvenes en familias madrileñas de origen extranjero. Tesis doctoral, Universidad Nacional de Educación a Distancia, Madrid.

Geronimi, E., Cachón, L. y Texidó, E., 2004, Acuerdos bilaterales de migración de mano de obra: Estudio de casos, Programa de Migraciones Internacionales, Oficina Internacional del Trabajo, Ginebra.

Gil Araujo, S., 2005, “Cartografías migratorias: migraciones internacionales en el marco de las relaciones Norte-Sur", en N. Zúñiga (coord.), La migración. Un camino entre el desarrollo y la cooperación, Centro de Investigación para la Paz, Madrid, pp..

Gil Araujo, S. y González-Fernández, T., 2014, "International Migration, Public Policies and Domestic Work. Latin American Migrant Women in the Spanish Domestic Work Sector", en Women's Studies International Forum, en prensa.

Gonzálvez, H, 2010, Migración colombiana, género y parentesco: La organización social de los cuidados, Tesis doctoral, Universidad de Granada, Granada. 
Gregorio Gil, C., 1998, Migración femenina. Su impacto en las relaciones de género, NARCEA S.A. Ediciones, Madrid.

Gregorio Gil, C. y Ramírez Fernández, A., 2000, “¿En España es diferente...? Mujeres inmigrantes dominicanas y marroquíes", en Papers, $\mathrm{n}^{\circ}$ 60, pp. 257-273.

Guarnizo, L. E., 2008, Londres Latina. La presencia colombiana en la capital británica, Universidad Autónoma de Zacatecas, Miguel Ángel Porrua, librero editor, México DF.

Guarnizo, L. E., 2006, "El estado y la migración global colombiana", en Migración y desarrollo, Primer semestre, pp. 79-101.

Guarnizo, L. E., 2003, "La migración transnacional colombiana: implicaciones teóricas y prácticas", en Memoria seminario sobre migración internacional colombiana y la conformación de comunidades transnacionales. Ministerio de Relaciones Exteriores, Colombia Nos Une, Bogotá, pp. 25-43.

Instituto Nacional de Estadística (INE), 2013a, España en cifras 2013, Madrid.

Instituto Nacional de Estadística (INE), 2013b, Notas de prensa, Cifras de Población a 1 de julio de 2013, Estadística de Migraciones, Primer semestre de 2013, Datos Provisionales.

Juliano, D., 2012, "Género y trayectorias migratorias en época de crisis", en Papers 97 (3), pp. 523-540.

Medina Villegas, M. C., 2009, Los ausentes están siempre presentes. Una aproximación interpretativa de la experiencia materno-filial transnacional entre España y Colombia, Tesis Doctoral, Universidad Complutense de Madrid, Madrid.

Mejía, W., 2010, "Panorama del retorno reciente de migrantes internacionales a Colombia", Ponencia presentada en VI Jornada Fundación Carolina: migración y desarrollo humano, Cátedra Europa-2010, Cartagena.

Micolta, A., 2007, "Inmigrantes colombianos en España. Experiencia parental e inmigración", en Revista Latinoamericana de Ciencias Sociales Niñez y Juventud, 5 (1), pp. 1-25.

Mojica, J. A., 2009, "Zona cafetera tiene 50 mil niños huérfanos, de padres vivos", en El Tiempo, 12 de enero de 2009.

Pajares, M., 2010, Inmigración y mercado de trabajo, Informe 2010, Observatorio para la Inmigración, Madrid. 
Parella, S., 2003, Mujer, inmigrante y trabajadora. La triple discriminación. Anthropos, Barcelona.

Parreñas, R., 2001, Servants of globalization: women, migration and domestic work, Stanford University Press, Stanford.

Pedone, C., Echeverri, M. M. y Gil Araujo, S., (en prensa), “Entre dos orillas. Cambios en las formas de organización de las familias migrantes latinoamericanas en España en tiempos de crisis global", en M. E. Zavala Castelo y V. Rosee, (Eds.), Las migraciones femeninas en América Latina y las transformaciones en las relaciones de género. Colegio de México, México DF.

Pedone, C. y Gil Araujo, S., 2008, "Maternidades transnacionales entre América Latina y el Estado español. El impacto de las políticas migratorias en las estrategias de reagrupación familiar", en C. Solé, S. Parella, L. Cavalcanti, (Coords.), Nuevos retos del transnacionalismo en el estudio de las migraciones, Ministerio de Trabajo e Inmigración, Subdirección General de Información Administrativa y Publicaciones, Madrid, pp. 151-176.

Pedone, C., 2010, "'Lo de migrar me lo tomaría con calma': representaciones sociales de jóvenes en torno al proyecto migratorio familiar", en A. García, M. E. Gadea y A. Pedreño (Eds.), Tránsitos migratorios: contextos transnacionales y proyectos familiares en las migraciones actuales, Universidad de Murcia, Murcia, pp.141-170.

Pedone, C., 2006, Estrategias migratorias y poder. Tú siempre jalas a los tuyos, Abya-Yala- PMCD, Quito.

Pedreño, A. y Castellanos Ortega, M. L., 2010, "En busca de un lugar en el mundo. Itinerarios formativos-laborales de los hijos e hijas de familias inmigrantes en el campo murciano", en Grupo Interdisciplinario de Investigadoras Migrantes (Coords.), Familias, niños, niñas y jóvenes migrantes. Rompiendo estereotipos, IEPALA Editorial, Madrid, pp. 107-122.

Puyana, Y., Motoa, J. y Viviel, A., 2009, Entre aquí y allá. Las familias colombianas transnacionales, UE, Universidad Nacional de Colombia, Fundación Esperanza, Bogotá.

Puyana, Y., Micolta, A. y Palacio, M. C., 2013, (Edit.), Familias colombianas y migración internacional: entre la distancia y la proximidad, Universidad Nacional de Colombia, Facultad de Ciencias Humanas, Centro de Estudios Sociales (CES), Bogotá. 
Rivas, A. M. y Gonzálvez, H., 2009, Familias transnacionales colombianas. Transformaciones y permanencias en las relaciones familiares y de género, Cataratas, Madrid.

Sanabria, H., 2008, Los inmigrantes colombianos en España: trayectoria y perspectivas, Documento de Trabajo, $n^{\circ} 35 / 2008$, Real Instituto Elcano, Madrid.

Santos Pérez, M. L., 2011, Mujeres ausentes y cabezas de familia: realidades sociales y económicas de las familias transnacionales constituidas entre Colombia y España, Serie Avances de Investigación, n 63, CeALCI - Fundación Carolina, Madrid.

Solé, C., Parella, S. y Cavalcanti, L., 2007, Los vínculos económicos y familiares transnacionales. Los inmigrantes ecuatorianos $y$ peruanos en España, Fundación BBVA, Madrid.

Sorensen, N. N. y Guarnizo, L.E., 2007, "La Vida de la Familia Transnacional a través del Atlántico: La Experiencia de la Población Colombiana y Dominicana Migrante en Europa", en Puntos de Vista $\mathrm{n}^{\circ}$ 9, Cuadernos del Observatorio de las Migraciones y la Convivencia Intercultural de la Ciudad de Madrid, pp. 7-28.

Suárez Navas, L., 2004, “Un nuevo actor migratorio: jóvenes, rutas y ritos juveniles transnacionales", Ponencia presentada en VIII Congreso de Inmigración: Menores y juventud, nuevos retos, 22-24 de abril de 2004, Almería.

UN-INSTRAW, OIM, 2007, Género y remesas. Migración colombiana del AMCO hacia España, Bogotá.

Unidad para la Atención y Reparación Integral a las Víctimas, 2014, Reporte General sobre Victimas. http://www.unidadvictimas.gov.co/index.php/sala-deprensa/noticias. 